\title{
Clinical efficacy of a two-year oral health programme for infants and toddlers in Singapore
}

\author{
Bien $\underline{L a i}^{1}$, BDS, MSc, Wee Kiat $\underline{T a n}^{1}$, BDS, MSc, Qing Shu $\underline{L u^{2}}$, BSc, PhD
}

INTRODUCTION Dental caries, which is prevalent in Singapore preschoolers, is a disease that has a major impact on children's health and places a high cost on the society and health services. Oral health programmes for young children implemented in some parts of the world have been shown to be effective in the prevention of dental caries. We aimed to examine the clinical efficacy of a two-year oral health programme for infants and toddlers in Singapore.

METHODS 90 children and their caregivers participated in the programme, and 64 children, who were 24 months older than the intervention group at the initial visit, were recruited as controls in a quasi-experimental study design. We evaluated the presence of severe early childhood caries (SECC) and $\mathrm{d}_{3} \mathrm{mfs}$ in the control group at the initial visit and in the intervention group after the completion of the two-year programme.

RESULTS Some children in the intervention (7.8\%) and control (31.3\%) groups ( $p<0.001$ ) had SECC (difference $23.5 \%$, $95 \%$ confidence interval $11 \%-36 \%)$. A higher percentage of children in the intervention group had $\mathrm{d}_{3} \mathrm{mfs}=0$ and habits associated with low risk for caries. The odds of SECC in the control group were three times higher than that for the intervention group, and the effect was significant $(p=0.037)$ after adjustment for other significant risk factors.

CONCLUSION The preventive oral health programme in Singapore was successful in reducing SECC among infants and toddlers when targeted behaviour modifications were implemented.

Keywords: dental caries, early dental visits, infant oral health, paediatric dentistry

\section{INTRODUCTION}

Dental caries remains the most common chronic childhood disease affecting children globally. ${ }^{(1-3)}$ Despite being one of the richest countries in the world, with a gross domestic product per capita of SGD 72,711 and a high literacy rate of $96.8 \%$, ${ }^{(4)}$ Singapore continues to struggle with early childhood caries. The implementation of the School Dental Service programme, aimed at preventing and treating dental caries in school-going children (age range 6-18 years), has proven to be extremely successful. Since its inception in 1949, we have seen a significant increase in the number of children (age range 6-11 years) with caries-free dentition, from $27.8 \%$ in 1970 to $58.2 \%$ in 1994, and a corresponding decline in the incidence of $\mathrm{dft}$ (d: decayed, f: filled due to decay, t: primary tooth) and DMFT (D: decayed, M: missing due to decay, F: filled due to decay, $\mathrm{T}$ : permanent tooth). ${ }^{(5)}$ The current DMFT value of 0.41 among 12-year-old children puts the country on par with other similar industrialised nations. ${ }^{(6)}$ However, recent studies have indicated a disproportionately high caries prevalence. One study reported a prevalence of $40 \%$ among 3-6-year-old children, with a racial and lower socioeconomic status predilection, ${ }^{(7)}$ while another study conducted among 18-48-month-old infants reported a caries prevalence of $48 \%{ }^{(8)}$ Early childhood caries can progress rapidly in deciduous dentition, resulting in pain and infection, affecting a child's nutritional intake, growth and development, and severely impacting their overall quality of life. ${ }^{(9)}$

Compounding the issue is the seemingly low oral health awareness among Singaporean parents. Despite the high prevalence of dental caries, only $8 \%$ of children aged $3-6$ years and $3 \%$ of children aged $18-48$ months received regular dental care. ${ }^{(7,8)}$ Only $1 \%$ of parents were aware of the age one dental visit recommendation by the American Academy of Pediatric Dentistry (AAPD). ${ }^{(8)}$ AAPD recommends that all children should have their first dental visit at no later than one year of age, with a heavy emphasis on preventive messages and anticipatory guidance. ${ }^{(10)}$ A delay in seeking dental care may result in increased severity of disease, leading to more extensive and costly treatments. ${ }^{(11)}$ In addition, general anaesthesia may be required to provide quality dental care for paediatric patients due to behavioural challenges, developmental immaturity and extensive treatment needs. The use of operating theatres for such elective procedures adds on to the cost burden of parents and the society. Early preventive care has the potential to decrease the number of emergency visits and cost of dental care. ${ }^{(11)}$

Early preventive dental care may be given through a non-invasive infant oral health programme, which includes a comprehensive oral examination, caries risk assessment, preventive services, and anticipatory guidance on diet, nonnutritive habits, growth and development, injury prevention and oral health practices. ${ }^{(10,12)}$ These programmes also maximise exposure to caries-reducing fluoride in a younger population, with fluoride varnish showing excellent effectiveness and safety profile. ${ }^{(13,14)}$ Infant oral health programmes implemented in several parts of the world have been shown to be effective in the prevention of dental caries. Children whose caregivers have attended oral health programmes had lower mean dmfs

${ }^{1}$ Paediatric Dentistry Unit, National Dental Centre Singapore, ${ }^{2}$ Department of Biostatistics, Singapore Clinical Research Institute, Singapore

Correspondence: Dr Lai Bien, Consultant, Paediatric Dentistry Unit, National Dental Centre Singapore, 5 Second Hospital Avenue, Singapore 168938. bien.lai.w.p@singhealth.com.sg 
(d: carious lesion; m: missing due to caries; $f$ : filled due to caries; s: surface) scores, ${ }^{(15,16)}$ lower caries prevalence ${ }^{(17-20)}$ and lower mean carious dental surfaces. ${ }^{(21-23)}$ In addition, children who had early preventive dental care incurred lower dental-related costs compared to those who received care later due to associated restorative costs. ${ }^{(11)}$ On the other hand, there were studies that reported minimal effects on caries reduction; ${ }^{(24,25)}$ which may be culturally related. The implementation of an infant oral health programme has the potential of impacting caries prevalence in the younger population, and perhaps even has long-term effects in older children. However, it requires support from the public health system in terms of collaboration with medical professionals, monetary resources and formal publicity. The objective of this exploratory study was to examine the clinical efficacy of an oral health programme for infants and toddlers in Singapore.

\section{METHODS}

A quasi-experimental design was employed for this study. From October 2012 to March 2013, eligible children were recruited from the National Dental Centre Singapore Paediatric Dentistry Clinic, nine primary health outpatient clinics (i.e. polyclinics), and the Singapore General Hospital Baby and Child Clinic. The study received full approval from the SingHealth Centralised Institutional Review Board Singapore (2012/482/D).

Children who were under 18 months of age at recruitment were enrolled in a two-year risk-based preventive oral health programme. At the completion of the two-year programme, outcome measures were evaluated for the intervention group. The quasi-experimental design entailed the recruitment of a matched older control group at their first visit, as it was deemed unethical to provide no intervention in a true prospective control group. Children in the control group were recruited at the same time and outcome measures were taken at enrolment, with no follow-up requirement.

The inclusion criteria for children in the intervention group were: (a) 0-18 months of age; (b) dentate with no congenital condition related to tooth structures; (c) in good health (American Society of Anesthesiologists physical status class I or II) and did not have special healthcare needs; (d) families who could commit to dental visits and planned to reside in Singapore for at least two years; (e) children with no previous dental experience; and (f) signed informed consent by parent or legal guardian. The inclusion criteria for children in the control group were similar, except for the age criterion being 24-42 months. A designated interpreter was provided for each of Singapore's three major second languages (i.e. Mandarin, Malay and Tamil) if the parent did not speak or read English. Children were excluded if they did not meet the above inclusion criteria or if their parents could not understand any of the four languages.

Each child was assigned to one of four calibrated paediatric dentists. All initial examinations for children in the intervention group were performed in the knee-to-knee position, with a welldirected light source from the dental unit in addition to the room light, using the mouth mirror and gauze to dry the teeth. When necessary, light active restraint by the caregiver was employed.
The caries diagnosis was based on visual criteria only. Intraoral radiographs were not taken. Cavitated and precavitated lesions were charted. A demineralised area of enamel with no loss of surface continuity was defined as a precavitated lesion. A cavitated lesion involved a loss of enamel surface continuity. The overall caries risk was assessed using the AAPD caries risk assessment tool for dental providers and based on the clinical judgement of the examiner. ${ }^{(10)}$

All children received a risk-based preventive programme comprising the following: (a) oral health education about the aetiology of dental caries, caries progression and prevention, and parental oral health; (b) anticipatory guidance on diet, oral health care practices, including tooth brushing and fluoride use, non-nutritional habits, trauma prevention, and growth and development; (c) topical fluoride varnish ( $5 \%$ sodium fluoride; Duraphat ${ }^{\circledR}$, Colgate, Waltrop, Germany), which was applied on all tooth surfaces for children who were considered at high caries risk; ${ }^{(10)}$ and (d) recommendation for dental review visits.

Children in the intervention group were scheduled for a dental review visit every six months for a period of two years, with the exception of those at high caries risk, who had additional appointments scheduled between their standardised six-month visits. Subsequent dental visits for children in the intervention group involved the same clinical procedure and the same outcome measures were recorded. The major components of the oral health programme were standardised, but the individual content of each component was customised to the child's history and clinical findings. Children who had treatment needs were referred for dental care. Children who were lost to follow-up or exceeded the window period of one month from the scheduled review visit date were excluded.

Severe early childhood caries (SECC) is defined as any sign of smooth-surface caries in children younger than three years old. For children in the $3-5$ years age group, $\geq 1 \mathrm{dmfs}$ in primary maxillary anterior teeth, or dmfs score $\geq 4$ (age three years), $\geq 5$ (age four years) or $\geq 6$ (age five years) surfaces constitutes SECC. ${ }^{(12)}$ The primary outcome measure was the presence of SECC (including precavitated lesions). The secondary outcome measure was the dmfs score. Behavioural changes in dietary habits, oral care practices and oral hygiene status were also compared. Although examiners were not aware of the study groups at the initial visit, blinding was not possible due to the difference in age.

A sample size of 64 patients per group was required to detect a difference of $20 \%$ in the proportion of patients with SECC between the two groups (10\% vs. $30 \%$ ) for $80 \%$ power at twosided $\alpha=0.05$. By factoring in a $40 \%$ attrition rate from previous studies at this institution, 107 patients and 64 controls were projected for recruitment. For examiner calibration, the intraoral photographs of ten de-identified children were used for interexaminer agreement among the four dentists who performed the outcome assessments. Dental caries and precavitated lesions were charted. For three patients, cases were re-charted to determine intra-examiner agreement.

Statistical analyses were performed using SAS version 9.3 (SAS Institute, Cary, NC, USA). Continuous variables were compared using independent sample $t$-test and Pearson's chi-square test, 
while Fisher's exact test was used for categorical variables, if appropriate. Comparison of outcomes was done using MannWhitney $U$-test for $\mathrm{d}_{3} \mathrm{mfs}\left(\mathrm{d}_{3}\right.$ : cavitated carious lesion; $\mathrm{m}$ : missing due to caries; f: filled due to caries; s: surface) and Pearson's chisquare test for the presence of SECC. Odds ratios of the presence of SECC and their 95\% confidence intervals (Cls) were calculated from logistic regression analyses. Inter- and intra-examiner agreements were analysed using Kappa statistic.

\section{RESULTS}

In all, 107 children were recruited for the intervention group and 64 for the control group. The attrition rate was $15.9 \%(n=17)$, with 90 children in the intervention group completing the study (Fig. 1). All children in the intervention group were caries-free at the initial visit. Demographic details of the study population are presented in Table I.

Compared to those in the intervention group, more children in the control group had habits associated with high caries risk, such as the presence of milk bottle habit and drinking milk to sleep (Table II). Moreover, caries preventive practices, such as the use of fluoridated toothpaste and tooth-brushing after last night feed, were more prevalent for children in the intervention group than those in the control group. In the study group, $37.8 \%$ of patients had good oral hygiene compared to $4.7 \%$ in the control group. More caregivers in the control group reported difficulties in brushing their child's teeth.

$31.3 \%$ of children in the control group had SECC compared to $7.8 \%$ in the intervention group (difference of $23.5 \%, 95 \%$
Cl 11\%-36\%; Table III). Regardless of age, the general trend showed a lower proportion of children having dental caries in the intervention group compared to the control group. Stratified by age group (age $<$ or $\geq 3$ years), the Cochran-Mantel-Haenszel estimate of odds ratio of SECC between the intervention and control groups was $0.32(95 \% \mathrm{Cl} 0.11-0.86 ; \mathrm{p}=0.015)$, and using the Breslow-Day test, there was no evidence of homogeneity of odds ratios between the age groups $(p=0.205)$. The majority of children in the intervention group (91.1\%) had $\mathrm{d}_{3} \mathrm{mfs}=0$ as compared to the control group $(84.4 \%)$. There was greater severity of dental caries in the control group, with a higher proportion of children having $\mathrm{d}_{3} \mathrm{mfs}>5$. The odds of children in the control group having SECC were three times higher than those in the intervention group after adjustment for potential confounders. The effect of parents' educational level remained significant after adjustment for other factors in the regression analysis (Table IV). Children whose parents did not attend university were nearly three times more likely to have SECC when compared to those whose parents had bachelor's degrees. The weighted Kappa coefficient for intra-examiner agreement was very good at $0.835(95 \% \mathrm{Cl} 0.725-0.772 ; \mathrm{p}<$ 0.0001). The Fleiss' Kappa of inter-examiner agreement for the four dentists was substantial at $0.748(95 \% \mathrm{Cl} 0.802-0.868 ; \mathrm{p}<0.0001)$.

\section{DISCUSSION}

There is a lack of clear evidence of the effectiveness of infant oral health programmes in different populations. In this exploratory study, we demonstrated that targeting infants and toddlers with oral preventive messages has the potential to significantly reduce

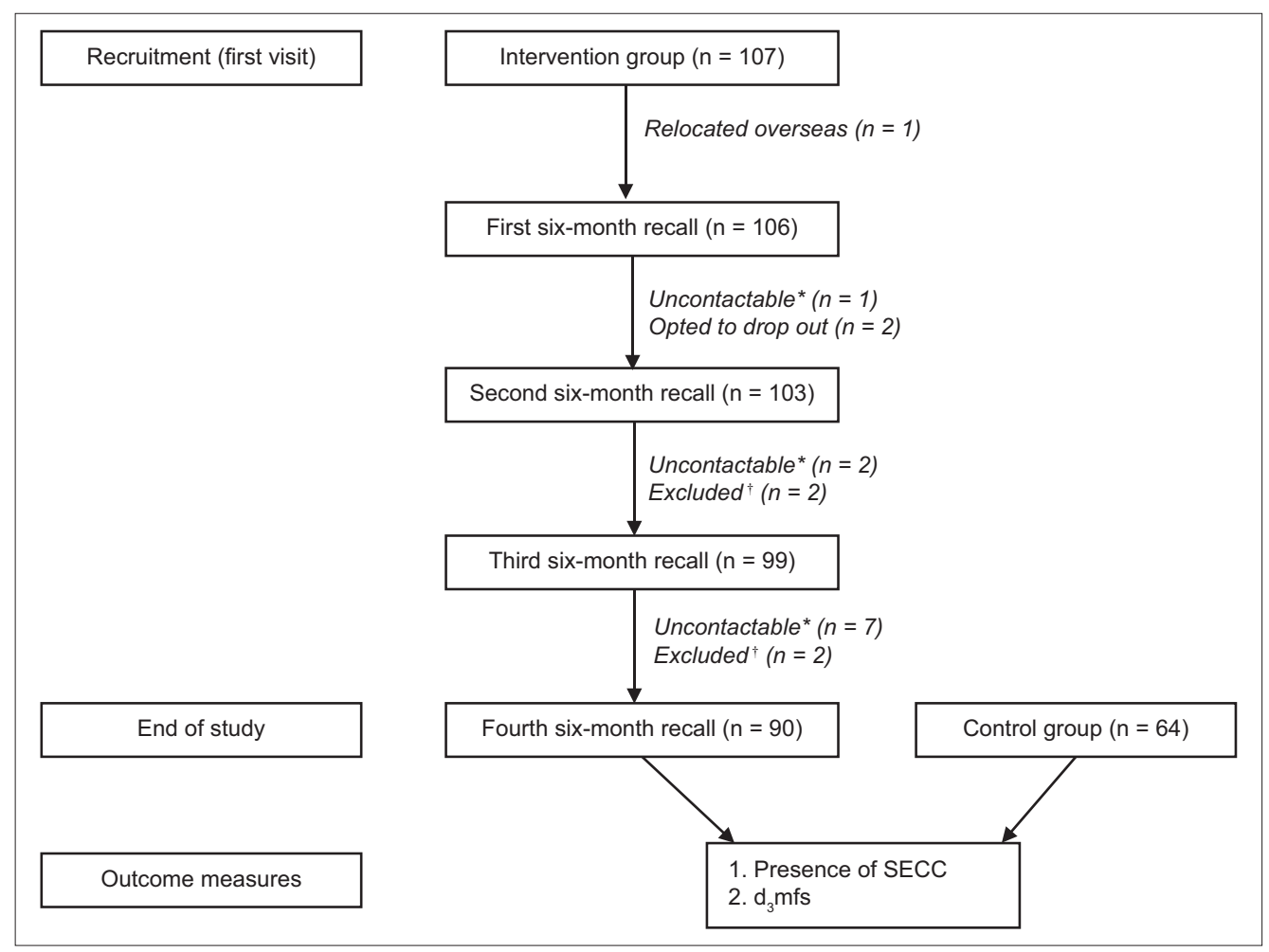

Fig. 1 Flowchart of participants shows the key stages of the study. *Three attempts (at different days and times over two weeks) were made to contact the caregiver of each child at the telephone numbers provided at the start of the study. +Children who were lost to follow-up exceeding the window period of one month from the scheduled recall visit date were excluded from the study. $d_{3} m f s$ : decayed, missing and filled tooth surfaces; SECC: severe early childhood caries 
Table I. Demographics of the study population.

\begin{tabular}{|c|c|c|c|}
\hline \multirow[t]{2}{*}{ Variable } & \multicolumn{2}{|c|}{ No. (\%) } & \multirow[t]{2}{*}{ p-value } \\
\hline & Intervention $(\mathrm{n}=90)$ & Control $(n=64)$ & \\
\hline Male gender & $45(50.0)$ & $34(53.1)$ & 0.702 \\
\hline Age (mth)* & $37.7 \pm 4.5$ & $30.8 \pm 5.9$ & $<0.0001$ \\
\hline Ethnicity & & & 0.041 \\
\hline Chinese & $83(92.2)$ & $52(81.3)$ & \\
\hline Monthly household income (SGD) & & & 0.049 \\
\hline$<4,000$ & $13(14.4)$ & $9(14.1)$ & \\
\hline $4,000-6,999$ & $24(26.7)$ & $25(39.1)$ & \\
\hline $7,000-10,999$ & $27(30.0)$ & $23(35.9)$ & \\
\hline$\geq 11,000$ & $26(28.9)$ & $7(10.9)$ & \\
\hline Caregiver's highest attained qualification & & & 0.040 \\
\hline Bachelor's degree and above & $65(72.2)$ & $36(56.3)$ & \\
\hline Caregiver type & & & 0.545 \\
\hline Mother & $71(78.9)$ & $53(82.8)$ & \\
\hline Other & $19(21.1)$ & $11(17.2)$ & \\
\hline Caregiver's last dental visit & & & 0.254 \\
\hline$\leq 6$ mths & $38(42.2)$ & $34(53.1)$ & \\
\hline$>6$ mths and $\leq 1 \mathrm{yr}$ & $23(25.6)$ & $9(14.1)$ & \\
\hline$>1 \mathrm{yr}$ & $22(24.4)$ & $18(28.1)$ & \\
\hline Cannot recall & $7(7.8)$ & $3(4.7)$ & \\
\hline
\end{tabular}

*Data presented as mean \pm standard deviation.

Table II. Findings on oral hygiene and questionnaire responses.

\begin{tabular}{|c|c|c|c|}
\hline \multirow[t]{2}{*}{ Variable } & \multicolumn{2}{|c|}{ No. (\%) } & \multirow[t]{2}{*}{ p-value } \\
\hline & Intervention $(\mathrm{n}=90)$ & Control $(n=64)$ & \\
\hline Oral hygiene & & & $<0.0001$ \\
\hline Good & $34(37.8)$ & $3(4.7)$ & \\
\hline Fair & $51(56.7)$ & $52(81.3)$ & \\
\hline Poor & $5(5.6)$ & $9(14.1)$ & \\
\hline History of on-demand breastfeeding* & $36(46.2)$ & $21(32.8)$ & 0.283 \\
\hline Juice intake & $82(91.1)$ & $32(50.0)$ & $<0.0001$ \\
\hline Snacking more than 3 times daily & $6(6.7)$ & $9(14.1)$ & 0.133 \\
\hline Drinking milk in a bottle to sleep & $5(5.6)$ & $15(23.4)$ & 0.001 \\
\hline Presence of milk bottle habit & $65(72.2)$ & $60(93.8)$ & $<0.001$ \\
\hline \multicolumn{4}{|l|}{ Fluoride use } \\
\hline Use of fluoridated toothpaste ${ }^{\dagger}$ & $82(94.3)$ & $22(38.6)$ & $<0.0001$ \\
\hline \multicolumn{4}{|l|}{ Oral hygiene practice } \\
\hline Tooth-brushing after last night feed ${ }^{\ddagger}$ & $65(78.3)$ & $10(19.6)$ & $<0.0001$ \\
\hline Difficulty in cleaning child's teeth & $23(25.6)$ & $32(50.0)$ & 0.002 \\
\hline
\end{tabular}

*Data available for 78 patients in the intervention group. +Data missing for 3 patients in the intervention group and 7 patients in the control group. $¥ D a t a$ missing for 7 patients in the intervention group and 13 patients in the control group.

caries prevalence. A 12-month cohort study of Singaporean preschool children ${ }^{(26)}$ reported a caries incidence of $44 \%$, as compared to our finding of $31.3 \%$ in the control group. In contrast, the 24 -month caries incidence of $7.8 \%$ in our intervention group was significantly lower. Our findings showed that our two-year, customised, risk-based preventive oral health programme was successful in reducing SECC. This echoes the findings of previous studies. ${ }^{(15,17-22)}$ Children who did not see a dentist at an early age were three times more likely to have SECC when compared to those who were enrolled in an infant oral health programme. This reinforces AAPD's recommendation of the age one dental visit.

The slightly lower prevalence in the control group could be attributed to our study sample having caregivers with higher income and educational level, and a lower percentage of participants of non-Chinese ethnicity. Although the educational level of caregivers and the household income of the families in 
this study were generally higher than those of studies from other countries, the household demographic in terms of education and income resembled that of the overall Singapore population. ${ }^{(27,28)}$

The success of the oral health programme was also demonstrated by the maintenance of good oral hygiene practices and fewer high caries risk habits in the intervention group. While milk may be considered less cariogenic than other sugarcontaining beverages, ${ }^{(29)}$ formula milk often contains added sugar. In addition, it is a common practice here for caregivers to sweeten milk with sugar or honey to make it more palatable to children. Despite the repeated advice to avoid bottle-feeding during the dental visits, the percentage of children in the intervention group having a bottle-feeding habit remained high at $72.2 \%$. Other studies have shown that not all children who had bottle-feeding habits exhibit SECC and not all children with significant dental caries had high-risk bottle-feeding habits. ${ }^{(30,31)}$ Indeed, $72.2 \%$ of our patients in the intervention group continued to use the milk bottle after two years of preventive dental visits, but only $7.8 \%$ had SECC. Caregivers could have found it more challenging to get their children to give up the milk bottle compared to brushing the child's teeth after the last feed. This is noteworthy for oral health providers, who should focus on changing modifiable behaviour that has the greatest impact on preventing caries. For example, $65.6 \%$ of caregivers in the control group used non-fluoridated toothpaste for their children. Considering that children's toothpaste

Table III. Outcome measures of the study.

\begin{tabular}{lccc}
\hline Variable & \multicolumn{2}{c}{ No. (\%) } & p-value \\
\cline { 2 - 3 } & $\begin{array}{c}\text { Intervention } \\
(\mathbf{n}=\mathbf{9 0})\end{array}$ & $\begin{array}{c}\text { Control } \\
(\mathbf{n}=64)\end{array}$ & \\
\hline Presence of SECC & $7(7.8)$ & $20(31.3)$ & $<0.001$ \\
Age < 3 yr & $4 / 22(18.2)$ & $16 / 49(32.7)$ & 0.210 \\
Age $\geq 3$ yr & $3 / 68(4.4)$ & $4 / 15(26.7)$ & 0.018 \\
$\mathbf{d}_{3}$ mfs score & & & 0.153 \\
Median (IQR) & $0.0(0.0)$ & $0.0(0.0)$ & \\
Min, Max & $0.0,23.0$ & $0.0,75.0$ & 0.031 \\
$\mathbf{d}_{3}$ mfs score by & & & \\
category & & & \\
0 & $82(91.1)$ & $54(84.4)$ & \\
$1-4$ & $6(6.7)$ & $2(3.1)$ & \\
$\geq 5$ & $2(2.2)$ & $8(12.5)$ & \\
\hline
\end{tabular}

$\mathrm{d}_{3} \mathrm{mfs}$ : decayed, missing and filled tooth surfaces; IQR: interquartile range; Max: maximum; Min: minimum; SECC: severe early childhood caries containing the desirable level of fluoride to protect against dental caries is widely available, the low number of children exposed to subtherapeutic levels of fluoride in their dentifrice was unacceptable. The use of toothpaste at the appropriate fluoride level according to caries risk should be emphasised.

Although dental caries is a preventable chronic disease, it remains prevalent in Singapore. Locally, awareness of the early preventive well-child visit is low among caregivers. Only $1 \%$ of parents were aware of the age one dental visit recommendation by AAPD. ${ }^{(8)}$ In this study, children who had attended early preventive dental visits had a lower proportion and lower severity of dental caries compared to their peers who had not accessed dental care. Given the success of the early preventive dental visit in Singapore, the next step would be to engage and collaborate with professionals whom young children see on a regular basis. AAPD recommends the first dental visit by age one. At one year old, children typically have more frequent encounters with medical and early childhood professionals compared to dental specialists. The targeted professionals would be early childhood educators, infant care caregivers, paediatricians, primary health caregivers (e.g. general practitioners), family medicine physicians, and nurses in polyclinics who vaccinate infants and toddlers. While physicians and nurses have extensive training in various aspects of paediatrics, training in oral health may be inadequate or missing in the current training curriculum. Increasing oral health awareness in these groups could help identify high-risk children and enable timely referrals to paediatric dentists. This collaboration could potentially reduce the dental caries rate and encourage preventive oral health practices at an early stage, before surgical intervention is necessitated. Future directions may include the incorporation of a section on oral health in the curriculum for Family Medicine and Paediatric Residency, or an oral health assessment on the checklist when children attend medical clinics for their 12-month vaccinations.

We acknowledge several limitations of this study. Firstly, despite efforts to conduct recruitment from different sites in the country, it is likely that caregivers who were more motivated, and presumably had higher education level and household income, would have signed up for the study. Therefore, the results may not be representative of the general Singapore population and must be interpreted with caution. Although the household income for both the intervention and control groups was comparable, only around $14 \%$ of each group came from families with household income

Table IV. Logistic regression analysis of severe early childhood caries.

\begin{tabular}{|c|c|c|c|c|}
\hline \multirow[t]{2}{*}{ Variable } & \multicolumn{2}{|c|}{ Unadjusted analysis } & \multicolumn{2}{|c|}{ Adjusted analysis } \\
\hline & OR $(95 \% \mathrm{CI})$ & p-value & OR $(95 \% \mathrm{Cl})$ & p-value \\
\hline Intervention vs. control group & $0.19(0.07-0.47)$ & $<0.001$ & $0.33(0.12-0.93)$ & 0.037 \\
\hline Age $^{*} \geq 3$ yr vs. $<3$ yr & $0.24(0.09-0.60)$ & 0.002 & $0.37(0.13-1.06)$ & 0.065 \\
\hline $\begin{array}{l}\text { Caregiver's highest attained qualification: bachelor's degree and above } \\
\text { vs. secondary/polytechnic }\end{array}$ & $0.23(0.10-0.56)$ & 0.001 & $0.27(0.10-0.69)$ & 0.007 \\
\hline Other ethnicities vs. Chinese ethnicity ${ }^{+}$ & $3.35(1.18-9.55)$ & 0.023 & $0.79(0.56-5.72)$ & 0.329 \\
\hline
\end{tabular}

*The interaction term between treatment group and age group was not statistically significant $(p=0.55)$. +Ethnicity was highly associated with caregiver's highest attained educational qualification, with Chinese participants having higher qualification than participants from other ethnic groups ( $p<0.0001$ ). Cl: confidence interval; OR: odds ratio 
less than SGD 4,000. Children from the other ethnic groups and those from low-income families were few in this study. A previous study of Singapore children did not find any association between socioeconomic status and poor homecare practices, but noted the association of poor homecare practices with ethnic groups for health behaviours. ${ }^{(26)}$ Despite the sample being skewed toward those of higher socioeconomic circumstance, $39.1 \%$ of children had SECC and $24.5 \%$ had dentinal caries at the end of our study across the two study groups. This suggests that dental caries is prevalent across all socioeconomic strata.

Secondly, it could be argued that the outcome measures in our study reflect the efficacy of fluoride varnish. Although the effectiveness of fluoride varnish in the prevention of dental caries has been proven, ${ }^{(13,14,32)}$ we felt that the essence of the infant oral health programme was the identification of highrisk children and the appropriate preventive interventions that follow. Thus, we measured the effect of an infant oral health programme with its essential components intact and included the optimal exposure to fluoride, which we found in the study to be woefully inadequate.

Thirdly, there was an age discrepancy of seven months between the two groups as a result of the accumulative delay of review visits. However, the intervention group still showed lower disease level, which emphasised the effectiveness of our intervention. Although the interaction term between the treatment group and age was not statistically significant ( $p=0.55$ ), we should have considered measures to better match the two groups so as to control possible confounding variables related to age. This may include stricter dental review visit schedules or recruiting the control group at a time closer to the last dental review visit of the intervention group.

Considering the above limitations, future research should focus on the following areas: (a) the study could be repeated on a larger scale, with a population that is more closely matched to the existing racial compositions in the country, and include families with low socioeconomic status; (b) with the proven efficacy of the oral health programme in this exploratory study, the cost effectiveness of the programme could be evaluated; (c) since the children in the current study were seen by paediatric dentists, there is a need to look at the training of manpower in order for the infant oral health programme to be rolled out at a national level; (d) we should examine access to oral healthcare and target individual barriers to care, such as operational issues involving referrals and follow-ups; and (e) the oral health literacy of caregivers in Singapore and its relation to dental caries in children should be evaluated, as our study findings suggest that caregivers' educational level may have an impact on overall preventive efforts.

In conclusion, the two-year preventive oral health programme for infants and toddlers was successful in reducing SECC when targeted behaviour modifications, such as reducing the consumption of sweetened milk and increased use of fluoridated toothpaste, were implemented. The result of this exploratory study provides preliminary clinical evidence for the training and allocation of manpower and the distribution of monetary resources for early caries prevention in children. It provides the basis for collaboration with medical and early childhood professionals, as well as the development of an infant oral health programme that can be tailored to the local population.

\section{ACKNOWLEDGEMENTS}

The authors would like to thank Ms Shen Guifeng and Ms Nurul Iryani for their administrative assistance; Dr Terry Teo and Dr Tabitha Chng for their assistance with data collection; and Miss Gita Krishnaswamy for providing the initial biostatistics consultation.

\section{REFERENCES}

1. Petersen PE. The World Oral Health Report 2003: continuous improvement of oral health in the $21^{\text {st }}$ century--the approach of the WHO Global Oral Health Programme. Community Dent Oral Epidemiol 2003; 31 Suppl 1:3-23.

2. Bagramian RA, Garcia-Godoy F, Volpe AR. The global increase in dental caries. A pending public health crisis. Am J Dent 2009; 22:3-8.

3. Department of Health and Human Services, National Institute of Dental and Craniofacial Research. Oral Health in America: A Report of the Surgeon General. Available at: http://www.nidcr.nih.gov/DataStatistics/SurgeonGeneral/ Documents/hck1ocv.@www.surgeon.fullrpt.pdf. Accessed January 14, 2017.

4. Department of Statistics, Singapore. National Account 2015. Available at: http:// www.singstat.gov.sg/statistics/latest-data \#1. Accessed May 4, 2016.

5. Lo GL, Bagramian RA. Declining prevalence of dental caries in school children in Singapore. Oral Dis 1997; 3:121-5.

6. Organisation for Economic Cooperation and Development. Health Status. Dental Health. Available at: http://stats.oecd.org/. Accessed May 17, 2016.

7. Gao XL, Hsu CY, Loh T, et al. Dental caries prevalence and distribution among preschoolers in Singapore. Community Dent Health 2009; 26:12-7.

8. Hong $\mathrm{CH}$, Bagramian RA, Hashim Nainar SM, et al. High caries prevalence and risk factors among young preschool children in an urban community with water fluoridation. Int J Paediatr Dent 2014; 24:32-42.

9. Sheiham A. Dental caries affects body weight, growth and quality of life in pre-school children. Br Dent J 2006; 201:625-6.

10. American Academy of Pediatric Dentistry. Guideline on caries-risk assessment and management of infants, children, and adolescents. Pediatr Dent 2013; 35:E157-64.

11. Savage MF, Lee JY, Kotch JB, Vann WF Jr. Early preventive dental visits: effects on subsequent utilization and costs. Pediatrics 2004; 114:e418-23.

12. American Academy of Pediatric Dentistry; American Academy of Pediatrics. Policy on early childhood caries (ECC): classifications, consequences, and preventive strategies. Pediatr Dent 2008-2009; 30(7 Suppl):40-3.

13. Marinho VC, Worthington HV, Walsh T, Clarkson JE. Fluoride varnishes for preventing dental caries in children and adolescents. Cochrane Database Syst Rev 2013; 7:CD002279.

14. Weintraub JA, Ramos-Gomez F, Jue B, et al. Fluoride varnish efficacy in preventing early childhood caries. J Dent Res 2006; 85:172-6.

15. Günay H, Dmoch-Bockhorn K, Günay Y, Geurtsen W. Effect on caries experience of a long-term preventive program for mothers and children starting during pregnancy. Clin Oral Investig 1998; 2:137-42.

16. Medeiros PB, Otero SA, Frencken JE, Bronkhorst EM, Leal SC. Effectiveness of an oral health program for mothers and their infants. Int J Paediatr Dent 2015; 25:29-34.

17. Gomez SS, Weber AA. Effectiveness of a caries preventive program in pregnant women and new mothers on their offspring. Int J Paediatr Dent 2001; 11:117-22.

18. Wennhall I, Mårtensson EM, Sjunnesson I, et al. Caries-preventive effect of an oral health program for preschool children in a low socio-economic, multicultural area in Sweden: results after one year. Acta Odontol Scand 2005; 63:163-7.

19. Neumann AS, Lee KJ, Gussy MG, et al. Impact of an oral health intervention on pre-school children $<3$ years of age in a rural setting in Australia. J Paediatr Child Health 2011; 47:367-72

20. Van den Branden S, Van den Broucke S, Leroy R, et al. Effect evaluation of an oral health promotion intervention in preschool children. Eur J Public Health 2014; 24:893-8.

21. Nurko C, Skur P, Brown JP. Caries prevalence of children in an infant oral health educational program at a WIC clinic. J Dent Child (Chic) 2003; 70:231-4.

22. Weinstein $P$, Harrison R, Benton T. Motivating parents to prevent caries in their young children: one-year findings. J Am Dent Assoc 2004; 135:731-8.

23. Minah G, Lin C, Coors S, et al. Evaluation of an early childhood caries prevention program at an urban pediatric clinic. Pediatr Dent 2008; 30:499-504.

24. Vachirarojpisan T, Shinada K, Kawaguchi Y. The process and outcome of a 
programme for preventing early childhood caries in Thailand. Community Dent Health 2005; 22:253-9.

25. Tubert-Jeannin S, Leger S, Manevy R. Addressing children's oral health inequalities: caries experience before and after the implementation of an oral health promotion program. Acta Odontol Scand 2012; 70:255-64.

26. Gao XL, Hsu CY, Xu YC, et al. Behavioral pathways explaining oral health disparity in children. J Dent Res 2010; 89:985-90.

27. Department of Statistics, Singapore. Population and Land Area 2015. Available at: http://www.singstat.gov.sg/statistics/latest-data\#16. Accessed May 4, 2016

28. Ministry of Health, Singapore. Population and Vital Statistics. Available at: https:// www.moh.gov.sg/content/moh_web/home/statistics/Health_Facts_Singapore/
Population_And_Vital_Statistics.html. Accessed April 4, 2016.

29. Reynolds EC, Riley PF, Storey E. Phosphoprotein inhibition of hydroxyapatite dissolution. Calcif Tissue Int 1982; 34 Suppl 2:S52-6.

30. O'Sullivan DM, Tinanoff N. Social and biological factors contributing to caries of the maxillary anterior teeth. Pediatr Dent 1993; 15:41-4.

31. Serwint JR, Mungo R, Negrete VF, Duggan AK, Korsch BM. Child-rearing practices and nursing caries. Pediatrics 1993; 92:233-7.

32. Bader JD, Shugars DA, Bonito AJ. A systematic review of selected caries prevention and management methods. Community Dent Oral Epidemiol 2001; 29:399-411. 